\title{
ESTRESSE OXIDATIVO COMO FATOR IMPORTANTE NA FISIOPATOLOGIA DA DOENÇA DE ALZHEIMER
}

GEMELLI, Tanise; ANDRADE, Rodrigo Binkowski de; CASTRO, Alexandre Luz. Biomédicos, doutorandos do Departamento de Bioquímica, Universidade Federal do Rio Grande do Sul, Porto Alegre-RS.

GARCIA, Larissa Pacheco. Médica, Universidade Federal de Ciências da Saúde de Porto Alegre-RS.

FUNCHAL, Cláudia. Farmacêutica bioquímica, mestre e doutora em Bioquímica, docente do Programa de Pós-Graduação em Biociências e Reabilitação do Centro Universitário Metodista do IPA. Rua Cel. Joaquim

Pedro Salgado, 80 - Porto Alegre-RS, CEP 90420-060. Tel: (51) 3316 1233. E-mail:

claudia.funchal@metodistadosul.edu.br; csfunchal@yahoo.com.br.

\section{Resumo}

O estresse oxidativo tem sido apontado como um ator importante na patogênese de várias doenças, incluindo doenças neurodegenerativas. A Doença de Alzheimer é uma desordem neurodegenerativa relacionada à idade, que é reconhecida como a forma mais comum de demência. Neste artigo, o objetivo foi revisar a participação de estresse oxidativo na Doença de Alzheimer. A Doença de Alzheimer é caracterizada histopatologicamente pela presença de placas amilóides extracelulares, emaranhados neurofibrilares intracelulares, a presença de oligômeros do peptídeo $\beta$-amiloide e a perda de sinapse. Além disso, o cérebro e o sistema nervoso são mais propensos ao estresse oxidativo e o dano oxidativo influencia nas doenças neurodegenerativas. Entretanto, o aumento do dano oxidativo, disfunção mitocondrial, acúmulo de agregados de proteínas oxidadas, inflamação e defeitos nas proteínas constituem patologias complexas interligadas que levam à morte celular de neurônios. Mutações no ácido desoxirribonucleico mitocondrial e estresse oxidativo contribuem para o envelhecimento, afetando diferentes sistemas de sinalização da célula, bem como conectividade neuronal, podendo levar à morte celular, sendo o maior fator de risco para doenças neurodegenerativas, como na Doença de Alzheimer.

Palavras-chave: Estresse Oxidativo; Doença de Alzheimer; doenças neurodegenerativas.

\section{OXIDATIVE STRESS AS AN IMPORTANT FACTOR In THE PATHOPHYSIOLOGY OF AlZHEIMER's Disease}

\begin{abstract}
Oxidative stress has been associated to play a crucial role in the pathogenesis of many diseases, including neurodegenerative diseases. Alzheimer's disease is an age-related neurodegenerative disorder, whichis recognized as the most common form of dementia. In this article, the aim was to review the involvement of oxidative stress on Alzheimer's disease. Alzheimer's disease is histopathologically characterized by the presence of extracellular amyloid plaques, intracellular neurofibrillary tangles, the presence of oligomers of amyloid-? peptide and loss of synapses. Moreover, the brain and the nervous system are more prone to oxidative stress and oxidative damage influences the neurodegenerative diseases. However, increased oxidative damage, mitochondrial dysfunction, accumulation of oxidized aggregated proteins, inflammation, and defects in proteins constitute complex intertwined pathologies that lead to neuronal cell death. Mitochondrial mutations on deoxyribonucleic acid and oxidative stress contribute to aging, affecting different cell signaling systems, as well as the connectivity and neuronal cell death may lead to the largest risk factor for neurodegenerative diseases such as Alzheimer's Disease.
\end{abstract}

KEYworDs: Oxidative stress; Alzheimer's Disease; Neurodegenerative diseases. 


\section{INTRODUÇão}

Em organismos aeróbicos saudáveis, há um equilíbrio entre a produção de várias espécies reativas e as defesas antioxidantes. Algumas dessas espécies não são imediatamente removidas, porque desempenham importantes papéis biológicos na defesa contra infecções e são também importantes mediadores da resposta inflamatória (HALLIWELL; GUTTERIDGE, 2007; HALLIWELL, 2011). O oxigênio é extremamente importante para a sobrevivência dos seres humanos, pois a vida aeróbica depende de combustão controlada para o fornecimento de energia. Esse processo é catalisado pela maquinaria metabólica, que pode ser danificada por descontroladas reações oxidativas associadas à produção de energia, levando a um aumento na produção de radicais livres e/ou espécies reativas (CHAVKO et al., 2003).

O termo radical livre é usado para determinar qualquer átomo ou molécula com existência independente, contendo um ou mais elétrons desemparelhado nas suas camadas de valência. Isso caracteriza uma atração para um campo magnético, o que pode torná-lo altamente reativo, capaz de reagir com qualquer composto situado próximo à sua órbita externa, tendo uma função oxidante ou redutora de elétrons (SHAMI; MOREIRA, 2004). Essas espécies são continuamente produzidas no organismo durante o metabolismo celular e podem desempenhar funções fisiológicas importantes, quando em concentrações baixas ou moderadas. As principais fontes de radicais livres são as organelas citoplasmáticas, que metabolizam o oxigênio, o nitrogênio e o cloro (HALLIWELL, 2006a).

Por causa das ameaças extremas de oxidação não controlada, a vida aeróbia desenvolveu um conjunto complexo de sistemas antioxidantes para controlar essas reações e reparar ou substituir a maquinaria celular danificada. Ao mesmo tempo, os sistemas enzimáticos evoluíram para a produção de espécies reativas, como moléculas de sinalização biológica, de reações biossintéticas, de defesa química e de desintoxicação (DEAN, 2006; HALLIWELL, 2011).

Nesse contexto, o estresse oxidativo é caracterizado como um desbalanço entre a produção de radicais livres e a capacidade da célula de se defender contra eles (HALLIWELL, 2006b). O alto consumo de oxigênio e o relativo baixo nível de antioxidantes resultam em suscetibilidade do tecido cerebral ao dano oxidativo (HALLIWELL, 2006a). Um aumento líquido nas espécies reativas pode produzir o dano a lipídios, proteínas e ao ácido desoxirribonucléico - DNA, induzindo à necrose ou à apoptose (KUSHNAREVA; NEWMEYER, 2010). Diferentes estudos mostram que reações oxidativas contribuem para muitas consequências do envelhecimento e dos principais processos patológicos, incluindo doenças cardiovasculares (STOCKER; KEANEY, 2004), doenças pulmonares (CANTIN, 2004), diabetes (BONNEFONT-ROUSSELOT, 2004), câncer (BERGER, 2005) e as doenças neurodegenerativas (VINA et al., 2004).

O crescimento de relatos clínicos e também experimentais sugere que o estresse oxidativo pode desempenhar um papel importante na degeneração neuronal, em doenças como a Doença de Alzheimer - DA, esclerose lateral amiotrófica, doença de Parkinson e doença de Huntington (MÖLLER, 2010; ALIEV et al., 2013). A perturbação na função ou a perda da integridade do retículo endoplasmático, devido ao estresse oxidativo, pode ser causada, por exemplo, pelo acúmulo e depósito de proteínas nãodobradas e por mudanças na homeostasia do cálcio dentro dessa organela (PASCHEN; FRANDSEN, 2001). Além disso, muitas evidências sugerem que as mitocôndrias tenham um papel central em doenças neurodegenerativas, em razão de seu papel na regulação da morte celular (MICHAEL et al., 2006). As mutações no DNA mitocondrial e o estresse oxidativo contribuem para o envelhecimento, sendo o maior fator de risco para doenças neurodegenerativas (MATTSON, 2006; ALIEV et al., 2013). A mitocôndria é a principal fonte de espécies reativas e de defesas antioxidantes nas células (CADENAS; DAVIES, 2000). Nessa organela são gerados os ânions superóxido $\left(\mathrm{O}_{2} \bullet\right)$ no espaço intermembrana pelo vazamento de elétrons, que se combinam com o oxigênio molecular no complexo 
III da cadeia de transporte de elétrons, em um processo que é dependente do potencial de membrana; e na matriz mitocondrial, através de um sítio não definido do complexo I (HAN et al., 2001). Além disso, a mitocôndria participa ativamente da homeostase de cálcio (PENG; JOU, 2010) e está envolvida em diversos processos que levam à morte celular, incluindo liberação de citocromo c (PENG; JOU, 2010; ALIEV et al.; 2011). Acredita-se que todos esses processos estejam interligados e que um desequilíbrio nessas funções possa estar envolvido na fisiopatologia de diversas doenças neurodegenerativas (VILA et al., 2008).

O estresse oxidativo foi implicado na progressão da DA, ocasionando a perda progressiva de populações de células neuronais específicas e associando-se com a formação de agregados de proteína, contribuindo para patogênese da doença (KEVIN et al., 2004; SULTANA et al., 2006; ; ALIEV et al., 2011).Portanto, o objetivo do nosso estudo foi realizar uma revisão da literatura sobre a DA e o estresse oxidativo.

\section{Metodologia}

Fizemos extensa revisão de literatura em bases de dados de artigos como Pubmed, Science Direct, Scopus e Scielo. A busca de artigos nessas bases de dados foi limitada nas línguas inglesa, espanhola e portuguesa. Também foram utilizados livros, dissertações de mestrado e teses de doutorado, e os artigos analisados foram selecionados por apresentarem grande pertinência ao tema. Foram selecionados artigos e livros entre 2000 e 2013 que discutiam estresse oxidativo, DA e que relacionavam doenças neurodegenerativas.

\section{Doença de Alzheimer}

ADA, uma doença neurodegenerativa progressiva, é a causa mais comum de síndrome demencial. É responsável por aproximadamente 60-70\% de todos os casos de demência. Sua prevalência aumenta com a idade, sendo de cerca de $1 \%$ no grupo etário de 60 64 anos, e 24-33\% naqueles com idade superior a 85 anos (APRAHAMIAN et al., 2009; SCHAEFFER et al., 2011). Sua apresentação mais comumé o déficit de memória, que envolve uma dificuldade em aprendizagem e na evocação de informações recentemente aprendidas. Esse sintoma evolui insidiosamente e progride lentamente ao longo do tempo. O declínio de outras funções, como a atenção, a linguagem e as funções executivas, pode surgir concomitantemente à alteração de memória ou posteriormente a ela (MEGA, 2002; GALLUCI et al., 2005; MCKHANN et al., 2011).

Dentre os principais fatores de risco correlacionados com a ocorrência da DA, situam-se a idade e a predisposição genética. Observa-se, entretanto, que a maioria dos casos de DA são esporádicos. Apenas 5\% dos pacientes têm a Doença de Alzheimer Familiar -DAF, uma forma incomum que tende a ocorrer mais cedo, e está relacionada com mutações em diversos genes, tais como o gene codificador da proteína precursora de amiloide - APP, da presinilina 1 (PS1) e 2 (PS2) e da apolipoproteína E (ApoE) (SHU; CHECLER, 2002; FRIDMAN et al., 2004; PHILIPSON et al., 2010; XIE et al., 2013).

Foram tambémapontados como fatores associados à DA a ocorrência de diabete melito e de hiperlipidemia, e ainda o uso de tabaco. Dentre os fatores protetores, estão a dieta mediterrânea, a atividade física, a escolaridade, a ingestão de ácido fólico e o consumo moderado de álcool. A qualidade da evidência para tais associações, porém, é baixa na literatura médica (SCARMEAS et al., 2009; MARTHA et al., 2011).

O diagnóstico de DA é baseado primariamente em características clínicas assentadas na anamnese e em um exame clínico, especialmente neurológico e psiquiátrico, detalhado. Exames laboratoriais e de neuroimagem devem ser utilizados no diagnóstico diferencial com outras causas de demência. O uso de biomarcadores, até o momento, é empregado unicamente em estudos experimentais (FROTA et al., 2011). As escalas, como o Mini-Mental State Examination - MMSE e o Clinical Dementia Rating - CDR, permitem avaliar a progressão dos sintomas (BLENNOW et al., 2006). O diagnóstico definitivo, entretanto, é obtido apenas após análise 
histopatológica de materiais de autópsia. Tal análise revela perda neuronal nas camadas piramidais do córtex cerebral e degenerações sinápticas intensas, tanto em nível hipocampal quanto neocortical, além de identificar os depósitos extracelulares do peptídeo beta-amiloide $(\beta \mathrm{A})$, as placas neuríticas ou senis e os emaranhados neurofibrilares - ENF intracelulares da proteína Tau hiperfosforilada (PERL, 2000; CHAUHAN; CHAUHAN, 2006; MARTHA et al., 2011). As placas senis são formadas através do acúmulo e da agregação do $\beta \mathrm{A}$, formando neuritos distróficos, astrócitos reativos e microglia ativada. Acredita-se que a concentração de placas neuríticas esteja relacionada ao grau de demência dos afetados. Os emaranhados neurofibrilares são formados pela deposição de proteína Tau, sendo sua principal função estabilizar os microtúbulos responsáveis em organizar a função neuronal (PERL, 2000; ALLARD et al., 2012).

Durante as últimas décadas, muitas hipóteses foram geradas para explicar a fisiopatologia da DA. A Hipótese da Cascata Amiloide considera como ponto chave no desenvolvimento da doença os efeitos neurotóxicos do $\beta$ A. O acúmulo das formas solúveis desse peptídeo leva à toxicidade sináptica e à neurotoxicidade, ocasionando a quebra da homeostase do cálcio, a indução de estresse oxidativo e a disfunção mitocondrial (BLENNOW et al., 2006; HAUPTMANN et al., 2006; KARRAN et al., 2011; ALIEV et al., 2013).

O $\beta$ Aé derivado por clivagem proteolítica da APP pela ação de beta e gama secretases. Em condições normais, o $\beta$ A é degradado por enzimas e retirado do encéfalo através de um balanço entre efluxo e influxo, mediados pela proteína receptora de LDL e receptores para produtos finais de glicosilação avançada. Na DA, um desbalanço entre a produção e o clearence do $\beta$ A levam ao depósito de oligômeros deste peptídeo (em especial $\beta$ A42) no espaço extracelular, ocasionando inibição do potencial de longa duração hipocampal e da plasticidade sináptica (BLENNOW et al., 2006; BUTTERFIELD et al., 2007; KARRAN et al., 2011). Estudos recentes revelam também a ocorrência de uma produção excessiva de $\beta$ A intraneuronal, podendo esta causar lise neuronal e formação de placas senis (FERNANDEZ-VIZARRA et al., 2004; KARRAN et al., 2011). As placas acabam por desencadear ativação astrocítica e microglial e a produção de resposta inflamatória, gerada no entorno dos depósitos de $\beta \mathrm{A}$, e assim contribuem com a morte de neurônios, com o déficit de neurotransmissores colinérgicos, serotoninérgicos, noradrenérgicos e com a redução dos níveis de somatostatina e corticotropina (ANGOSTO; GONZÁLEZ, 2009).

Uma teoria recente propõe que aAPPe a presinilina 1 (PS1) podem modular os sinais intracelulares e, assim, induzir anormalidades no ciclo celular, levando à morte neuronal e, possivelmente, à deposição de amiloide. Ahipótese é apoiada pela presença de uma rede complexa de proteínas, claramente envolvida na regulação de mecanismos de transdução de sinal que interagem com APP e PS1 (NIZZARI et al., 2012; XIE et al., 2013). Quando a sinalização ativada por essas proteínas se torna disfuncional nos neurônios, ocorre a ativação da via da enzima regula por sinal extracelular (ERK1/2), levando à neurodegeneração. Essa enzima está relacionada a estímulos extracelulares para a proliferação celular, a sobrevivência e a diferenciação, assim como para a morte celular e para a apoptose (NIZZARI et al., 2012).

\section{DOENÇA DE AlZHEIMER E ESTRESSE OXIDATIVO}

O estresse oxidativo na DA se manifesta através da presença de proteínas oxidadas, de produtos de glicosilação avançada, da peroxidação lipídica e da formação de espécies tóxicas, tais como peróxidos, álcoois, aldeídos, carbonilas, cetonas e de modificações oxidativas no DNA nuclear e mitocondrial (Figura 1) (GELLA et al., 2009). 


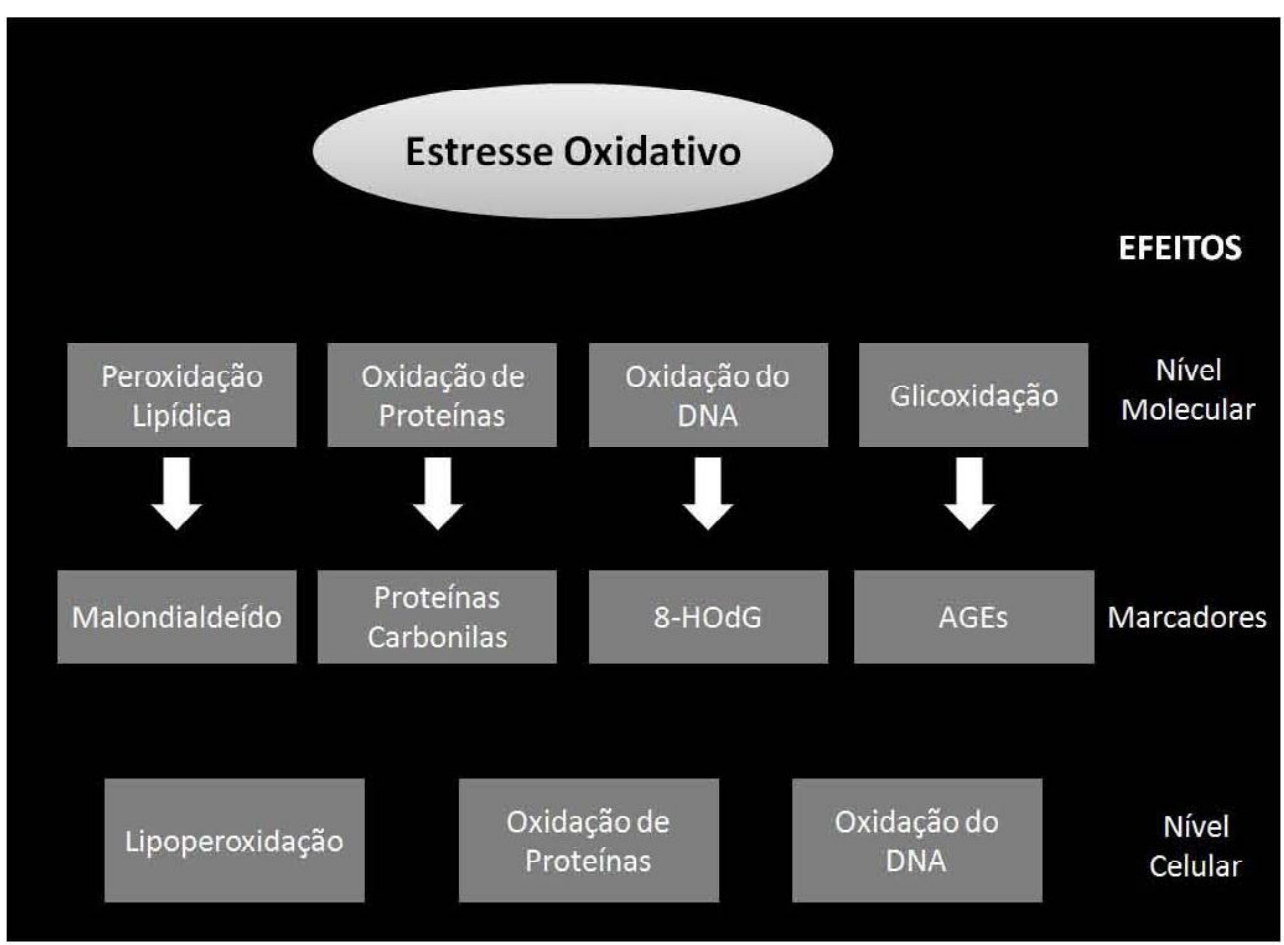

Figura 1 - Efeitos do estresse oxidativo sobre níveis moleculares e celulares e seus respectivos marcadores. 8-HOdG: 8-hidroxi-2 desoxiguanosina, AGEs: produtos finais da glicosilação avançada.

Fonte: Dados de pesquisa.

$\mathrm{Na}$ mitocôndria, o A reduz o potencial de membrana mitocondrial, inibe a cadeia transportadora de elétrons, diminui a taxa respiratória e induz a liberação de espécies reativas, favorecendo a ocorrência de apoptose (Figura 2) (ALIEV et al., 2013). O $\beta$ A pode também causar neurotoxicidade por meio da produção direta destas espécies reativas, através da interação com $\mathrm{Cu}^{2+}, \mathrm{Fe}^{2+}$ e $\mathrm{Zn}^{2+}$ (FINEFROCK et al., 2003; ALIEV et al.; 2011). Na presença de tais metais de transição, observa-se que o peróxido de hidrogênio é cataliticamente convertido em radical hidroxila de alta toxicidade, pelas reações de Fenton e Haber-Weiss (PÉREZ; ARANCIBIA, 2007). 


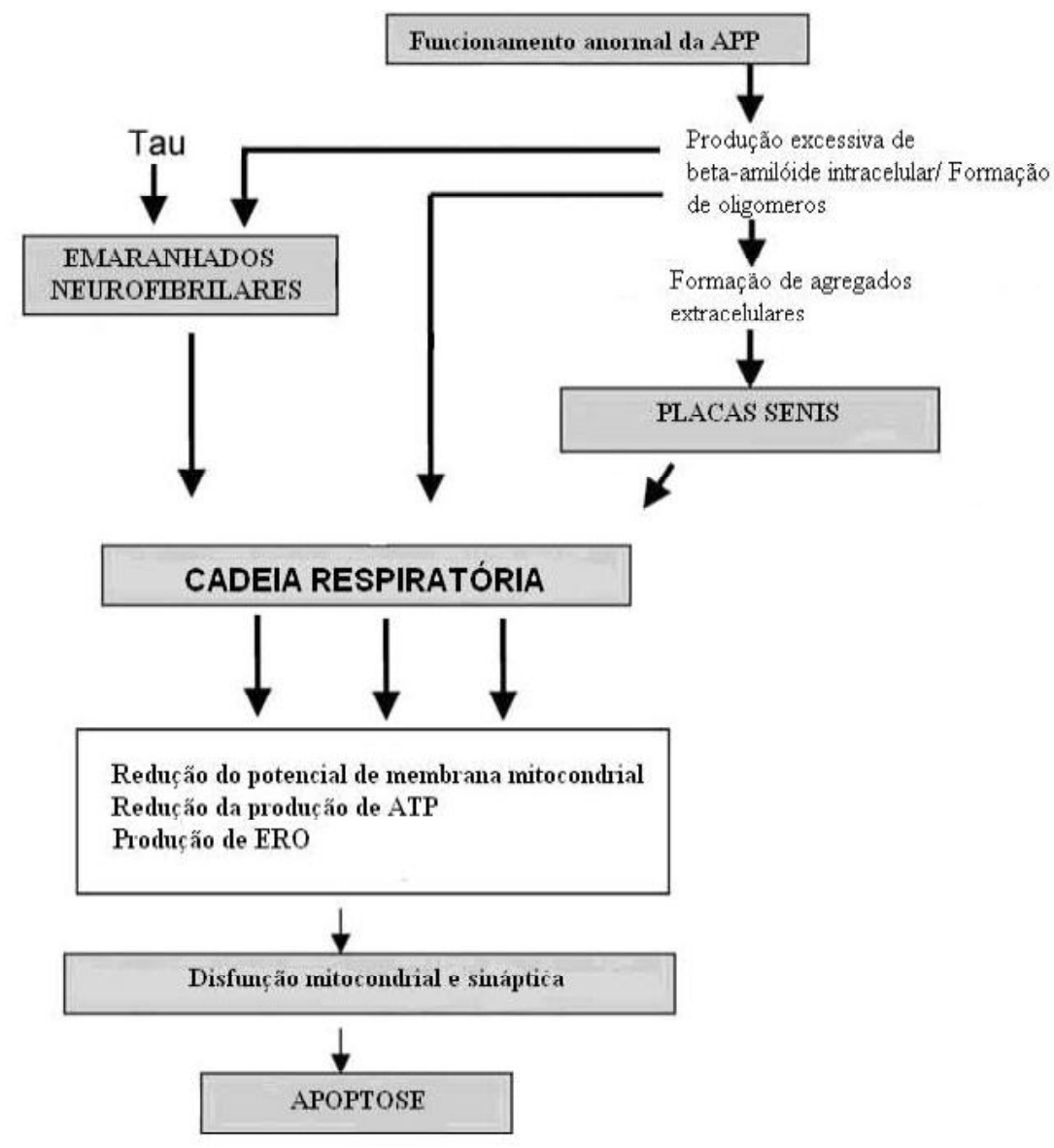

Figura 2 - Disfunção mitocondrial em DA. APP: precursor da proteína amilóide, ERO: espécies reativas de oxigênio, ATP: adenosina trifosfato, TAU: proteína TAU.

Fonte: Dados de pesquisa.

Observa-se também que o $\beta A$ se encontra fortemente relacionado ao dano oxidativo às membranas lipídicas, tornando-as mais hidrofílicas e alterando a função de transportadores, enzimas e receptores celulares localizados nessas membranas. Em pacientes com DA, observou-se um aumento na produção 4-hidroxi-2-trans-nonenal (4HNE), um dos principais produtos da oxidação lipídica, ocasionando modificações oxidativas do transportador de glutamato GLT1 e, assim, contribuindo para a neurodegeneração. Além disso, sabe-se que o 4 HNE se liga às proteínas glutationa-S-transferase e proteína 1 , ocasionando redução da atividade das mesmas (PÉREZ; ARANCIBIA, 2007).
Em estudos prévios, foi demonstrado que, através da ação das ERO, os lipídios de membrana tiveram sua conformação alterada e participaram da formação de emaranhados neurofibrilares em encéfalos de pacientes portadores de DA (ANSARI; SCHEFF, 2010; CHAUHAN; CHAUHAN, 2006; XIE et al., 2013).

O aumento de ERO na DA está relacionado com a oxidação de cadeias laterais de proteínas. Os grupos carbonilas são introduzidos nas proteínas pela oxidação da hidroxila dos aminoácidos de cadeia lateral dentro de derivados de aldeídos e cetonas, levando à carbonilação dessas proteínas. Esse grupamento também pode ser introduzido nas proteínas por 
oxidação direta dos aminoácidos lisina, arginina, prolina e treonina, ou a partir da clivagem de ligações peptídicas pela via $\alpha$-amidação ou pela oxidação dos resíduos de glutamil (GELLA et al., 2009). A produção de derivados reativos de carbonilas e aldeídos pode ser resultado da oxidação de lipídios, do DNA e de açúcares, que podem, por sua vez, reagir com proteínas e também formar carbonilas proteicas (VINA et al., 2004; HALLIWELL, 2011).

Estudos recentes demonstraram um aumento na quebra do DNA em cérebro de pacientes com DA. Esse achado pode ser explicado pelo aumento de ERO no cérebro, induzindo ao influxo de cálcio via receptores de glutamato, desencadeando uma toxicidade cerebral. Esse processo pode culminar com a morte celular (BUTTERFIELD et al., 2007). A oxidação do DNA é marcada pelo aumento dos níveis de 8-hidroxiguanosina (8-OHG), 8-hidroxiadenina, 5hidroxiuracila e 8-hidroxi-2 desoxiguanosina (8OHdG) no lobo temporal, parietale frontal na DA. Um hipótese para esse dano é o aumento de carbonilas livres nos núcleos de neurônios e glia na DA (LOVELL; MARKESBERY, 2007).

Com o passar dos anos, houve um aumento na pesquisa terapêutica, com importantes avanços quanto à utilização de nutrientes antioxidantes que podem reduzir o risco para a DA. A abordagem eficaz baseada em nutrição é de grande benefício a esses pacientes, devido a um risco relativamente baixo de efeitos colaterais, uma vez que medicamentos disponíveis para o tratamento DA possuem baixos efeitos e não alteram a progressão da doença. Alguns estudos epidemiológicos demonstraram que a dieta mediterrânea, rica em vegetais, azeite e peixe, que inclui também quantidades moderadas de vinho e baixas quantidades de carne vermelha, pode influenciar positivamente na DA (SCARMEAS et al., 2009; KNOPMAN, 2009). A utilização de nutrientes antioxidantes para retardar a progressão da DA é muito benéfica, pois o uso de compostos vitamínicos, como a vitamina De complexo B, especialmente a vitamina B12, estão associados como o declínio cognitivo no envelhecimento (ANNWEILER et al., 2010; NELSON et al., 2009; SOZIO et al., 2013).
Outro composto antioxidante com propriedades anti-amiloidogênicos é o resveratrol, no entanto, a aplicação terapêutica dos seus efeitos benéficos pode ser limitada. Frozza et al. (2013) demonstraram que a ação do resveratrol, quando administrado na forma de nanocápsula, pode aumentar significativamente a eficácia desse antioxidante em controlar parcialmente os efeitos deletérios do $\beta$ A.

Entretanto, abordagens baseadas em nutrição merecem uma investigação mais aprofundada, como parte integrante de estratégias terapêuticas, mas podem ser consideradas, em razão de seu baixo risco de efeitos colaterais e seu potencial para influenciar nos processos patológicos desde os primórdios do estabelecimento da DA.

\section{Conclusão}

Em razão de sua alta taxa metabólica, sua alta utilização de oxigênio, seu alto teor de ácidos graxos poliinsaturados oxidáveis e da presença de metais redox-ativos, bem como da capacidade relativamente reduzida de regeneração celular, o cérebro é particularmente vulnerável ao dano oxidativo (VALKO, 2007). Na DA foram relatados diferentes danos causados por espécies (XIE et al., 2013; ALIEV et al.; 2011). Marcadores de peroxidação lipídica, incluindo 4 hidroxinonenal (4-HNE) e malondialdeído (MDA), têm sido identificados no córtex cerebral e no hipocampo de pacientes com DA. Um sinal muito comum nas doenças neurodegenerativas é o acúmulo e depósito de proteínas deformadas oxidativamente que afetam diferentes sistemas de sinalização da célula, bem como afetam a conectividade neuronal, podendo levar à morte celular (FINEFROCK et al., 2003). Portanto, o estresse oxidativo parece desempenhar um importante papel no desenvolvimento de características presentes nas doenças neurodegenerativas, como na DA.

\section{REFERÊNCIAS}

ALIEV, G.; LI, Y.; PALACIOS, H. H.; OBRENOVICH, M. E. "Oxidative stress induced 
mitochondrial DNA deletion as a hallmark for the drug development in the context of the cerebrovascular diseases." Recent. Pat.

Cardiovasc. Drug. Discov., v. 6, p. 222-241, 2011.

ALIEV, G.; OBRENOVICH, M. E.; TABREZ, S.; JABIR, N. R.; REDDY, V. P.; LI, Y.; BURNSTOCK, G.; CACABELOS, R.; KAMAL, M. A. Link between Cancer and Alzheimer Disease via Oxidative Stress Induced by Nitric OxideDependent Mitochondrial DNA Over proliferation and Deletion. Oxid. Med. Cell. Longev. doi: 10.1155/962984, 2013.

ALLARD, S.; LEON, W. C.; PAKAVATHKUMAR, P.; BRUNO, M. A.; RIBEIRO-DA-SILVA, A.; CUELLO, A. C. "Impact of the NGF maturation and degradation pathway on the cortical cholinergic system phenotype." J. Neurosci. v. 32, p. 2002-2012, 2012.

ANSARI, M.A.; SCHEFF, S.W. Oxidative stress in the progression of Alzheimer disease in the frontal cortex. J. Neuropathol. Exp. Neurol. v. 69, p. 155-67. 2010.

ANNWEILER, C.; SCHOTT, A.M.; ROLLAND, Y.; BLAIN, H.; HERRMANN, F.R.; BEAUCHET, $\mathrm{O}$. Dietary intake of vitamin $\mathrm{D}$ and cognition in older women: a large population-based study. Neurology, v. 75, p. 1810-6, 2010.

AMES, B. N.; SHIGENAGA, M. K.; HAGEN, T. M. Oxidants, antioxidants, and the degenerative diseases of aging. Proc. Natl.Acad. Sci., v. 90, p. 7915-7922, 1993.

ANGOSTO, M. C.; GONZÁLEZ, P. G. Factores implicados en la patogénesis de la enfermedad de Alzheimer. Estrés oxidativo. 2009. 417-466. Monografías de la Real Academia Nacional de Farmacia. Madrid, 2009.
APRAHAMIAN, I.; MARTINELLI, J. E.; YASSUDA, M. S. Alzheimer's disease: an epidemiology and diagnosis review. Rev. Bras. Clin. Med., v. 7, p. 27-35, 2009.

BLENNOW, K.; DE LEON, M. J.;

ZETTERBERG, H. Alzheimer's disease. Lancet, v. 368, p. 387-403, 2006.

BOILLÉE, S.; VELDE, C. V.; CLEVELAND, D. W. ALS: A Disease of Motor Neurons and Their Nonneuronal Neighbors. Neuron., v. 52, p. 39-59, 2006.

BERGER, M. M. Can oxidative damage be treated nutritionally? Clin Nutr, v. 24, p. 172-183, 2005.

BONNEFONT-ROUSSELOT, D. The role of antioxidant micronutrients in the prevention of diabetic complications. Treat. Endocrinol, v. 3, p. 41-52, 2004.

BUTTERFIELD, D. A.; REED, T.; NEWMAN, F. S.; SULTANA, R. Roles of amyloid ?-peptideassociated oxidative stress and brain protein modifications in the pathogenesis of Alzheimer's disease and mild cognitive impairment. Free Radic. Biol. Med., v. 43, p. 658-677, 2007.

CADENAS, E.; DAVIES, K. J. A. Mitochondrial free radical generation, oxidative stress, and aging. Free Rad. Biol. Med., v. 29, p. 222-230, 2000.

CANTIN, A. M. Potential for antioxidant therapy of cystic fibrosis. Curr. Opin. Pulm. Med., v. 10, p. 531-536, 2004.

CHAUDHURI, K. R.; SCHAPIRA, A. H. Nonmotor symptoms of Parkinson's disease: dopaminergic pathophysiology and treatment. Lancet. Neurol., v.8, p.464-474, 2009.

CHAUHAN, V.; CHAUHAN, A. Oxidative stress in alzheimer's disease. Pathophysiology, v. 13, p. 
195-208, 2006.

CHAVKO, M.; AUKER, C. R.; MCCARRON, R. M. Relationship between protein nitration and oxidation and development of hyperoxic seizures.

Nitric. Oxide., v. 9, p. 18-23, 2003.

DEAN, P.; JONES. Redefining Oxidative Stress. Antioxid. Redox Signal, v. 8, p. 1865-1879, 2006.

KARRAN, E.; MERCKEN, M.; DE STROOPER, $B$. The amyloid cascade hypothesis for Alzheimer's disease: an appraisal for the development of therapeutics. Nat. Rev. Drug Discov., v. 10, p. 698-712, 2011.

FERNANDEZ-VIZARRA, P.; FERNANDEZ, A. P.; CASTRO-BLANCO, S.; SERRANO, J.; BENTURA, M. L.; MARTINEZ-MURILLO, R.; MARTINEZ, A.; RODRIGO, J. Intra- and extracellular Abeta and PHF in clinically evaluated cases of Alzheimer's disease. Histol. Histopathol. v. 19, p. 823-844, 2004.

FINEFROCK, A. E.; BUSH, A. I.; DORCISWAMY, P. M. Current status of Metals as Therapeutic Targets in Alzheimer's Disease. J. AM. Geriatr. Soc., v. 51, p. 1143-1148, 2003.

FOLSTEIN, S. Huntington's disease: a disorder of families. Baltimore: The Johns Hopkins University Press, P. 251, 1989.

FRIDMAN, C; GREGORIO, S. P.; DIAS NETO, E.; OJOPI, E. P. B. Alterações genéticas na doença de Alzheimer. Rev. psiquiatr. Clín., v.31, n.1, p. 19-25, 2004.

FROTA, N. A. F.; NITRINI, R.; DAMASCENO, B.P.; FORLENZA, O.V.; DIAS-TOSTA, E.; SILVA, A.B.; HERRERAJR, E. MAGALDI, R.M. Criteria for the diagnosis of Alzheimer's disease: Recommendations of the Scientific Department of Cognitive Neurology and Aging of the Brazilian
Academy of Neurology. Dement. Neuropsychol., v. 5(3), p. 146-152, 2011.

FROZZA, R.L.; BERNARDI, A.; HOPPE, J.B.; MENEGHETTI, A.B.; MATTÉ, A.; BATTASTINI, A.M.; POHLMANN, A.R.; GUTERRES, S.S.; SALBEGO, C. Neuroprotective effects of resveratrol against $\mathrm{A}$ ? administration in rats are improved by lipid-core nanocapsules. Mol Neurobiol, v. 47, p. 1066-80, 2013.

GALLUCCI, N. J.;TAMELINI, M. G.; FORLENZA, O. V. Diagnóstico Diferencial das Demências. Rev. Psiq. Clín., v. 32, p. 119-130, 2005.

GELLA, A. AND DURANY, N. Oxidative stress in Alzheimer disease. Cell Adh. Migr., v. 3, p. 88-93, 2009.

HALLIWELL, B. Proteasomal dysfunction: a common feature of neurodegenerative diseases? Implications for the environmental origins of neurodegeneration. Antioxid. Redox Signal, v. 8, p. 2007-2019, 2006a.

HALLIWELL, B. Reactive Species and Antioxidants. Redox Biology Is a Fundamental Theme of Aerobic Life. Plant. Physiology, v. 141, p. 312-322, 2006 b.

HALLIWELL, B. Free radicals and antioxidants quo vadis? Trends Pharmacol Sci., v. 32, p. 12530, 2011.

HALLIWELL, B.; GUTTERIDGE, J. M. C. Measurement of reactive species. In: Halliwell, B.; Gutteridge, J. M. C. Free Radic. Biol. Med. 4 ed. Oxford University Press, Oxford, 2007, p. 268-340.

HAN, D.; WILLIAMS, E.; CADENAS, E. Mitochondrial respiratory chain'dependent generation of superoxide anion and its release into the intermembrane space. Biochem. J., v. 
353, p. 411-416, 2001.

HAUPTMANNM , S.; KEIL, U.; SCHERPING, I.; BONERT, A.; ECKERT, A.; MULLER, W. E. Mitochondrial dysfunction in sporadic and genetic Alzheimer's disease. Exp. Gerontol., v. 41, p. 668673, 2006.

HUGHES, A. J.; DANIEL, S. E.; KILFORD, L.; LEES, A. J. Accuracy of clinical diagnosis of idiopathic Parkinson's disease: a clinico-pathological study of 100 cases. J. Neurol. Neurosurg.

Psychiatry, v. 55, p.181-184, 1992.

KEVIN, J.; BARNHAM, C. L.; MASTERS AND ASHLEY, I.; BUSH. Neurodegenerative diseases and oxidative stress. Nat. rev., v. 3, p. 205-214, 2004.

KNOPMAN, D.S. Mediterranean diet and late-life cognitive impairment: a taste of benefit. JAMA, v. 302, p. 686-687, 2009.

KUSHNAREVA, Y, NEWMEYER, D.D.

Bioenergetics and cell death. Ann. N. Y.Acad. Sci. v. 1201 p. $50-7.2010$.

LIU, X.; KIM, C. N.; YANG, J.; JEMMERSON, R.; WANG, X. Induction of apoptotic program in cell'free extracts:

requirement for dATP and cytochrome c. Cell., v. 86, p. 147-157, 1996.

LOVELL, A. M. AND MARKESBERY, W. R. Oxidative DNA damage in mild cognitive impairment and late-stage Alzheimer's disease. Nucleic Acids Res., v. 35, p. 7497-7504, 2007.

MARTHA, L.; DAVIGLUS M. L.; PLASSMAN B.L.; PIRZADAAA.;BELL, C.C.; BOWEN P.E; BURKE, J.R.; CONNOLLY JR. E.S.; DUNBARJACOB J. M.; GRANIERI, E.C.; MCGARRY, K.; PATEL D.;TREVISAN M.; WILLIAMS JR,J.W. Risk Factors and Preventive Interventions for
Alzheimer Disease: State of the Science. Arch.

Neurol., v. 68, p. 1185-1190, 2011.

MATTSON, M. P. Neuronal life-and-death signaling, apoptosis, and neurodegenerative disorders. Antioxid. Redox Signal, v. 8, p.19972006, 2006.

MEGA, M. S. Differencial diagnosis of dementia: clinical examination and laboratory assessment. Clin. Cornerstone, v. 4, p. 53-65, 2002.

MCKHANN, G.M.; KNOPMAN, D.S.; CHERTKOW, H.; HYMAN, B.T.; JACK, C.R.; KAWAS, C.H.; KLUNK, W.E.; KOROSHETZ, W.J.; MANLY, J.J.; MAYEUX, R.; MOHS, R.C.; MORRIS, J.C.;. ROSSOR, M.N.; SCHELTENS, P.; CARRILLO, M.C.; THIES, B.; WEINTRAUB, S.; MEGA, M. S. Differencial diagnosis of dementia: clinical examination and laboratory assessment. Clin. Cornerstone, v. 4, p. 53-65, 2002.

MICHAEL, T.; LIN, M.; FLINT, BEAL.

Mitochondrial dysfunction and oxidative stress in neurodegenerative diseases. Nature, v. 443, p. 787 795, 2006.

NELSON, C.; WENGREEN, H.J.; MUNGER, R.G.; CORCORAN, C.D. Dietary folate, vitamin B12, vitamin B-6 and incident Alzheimer's disease: The cache county memory, health and aging study. J Nutr Health Aging, v. 13, p. 899-905, 2009.

NICHOLLS, D. G; AKERMAN, K. E. O. Mitochondrial calcium transport. Biochim. Biophys. Acta., v. 683, p. 57-88, 1982.

NIZZARI, M.; THELLUNG, S; CORSARO, A.; VILLA, V.; PAGANO, A.; PORCILE, C.; RUSSO, C.; FLORIO, T. Neurodegeneration in Alzheimer Disease: Role of Amyloid Precursor Protein and Presenilin 1 Intracellular Signaling. J. Toxicol., 2012. Published online. 
PASCHEN, W.; FRANDSEN, A. Endoplasmic reticulum dysfunction a common denominator for cell injury in acute and degenerative diseases of the brain? J. Neurochem., v. 79, p. 719-725, 2001.

PÉREZ, M. A.; ARANCIBIA, S.R. Estrés oxidativo y neurodegeneración: ¿causa o consecuencia? Arch. Neurocien., v. 12, p. 45-54, 2007.

PERL, D. P. Neuropathology of Alzheimer's disease and related disorders. Neurol. Clin., v. 18, p. 847, 2000 .

PENG, T.I.; JOU, M.J. Oxidative stress caused by mitochondrial calcium overload. Ann N YAcad Sci. v. 8, p. 1201-183, 2010.

PHELPS, C.H. The diagnosis of dementia due to Alzheimer's disease: Recommendations from the National Institute on Aging-Alzheimer's Association workgroups on diagnostic guidelines for Alzheimer's disease. Alzheimers Dement. v. 7(3), p.263-269, 2011.

PHILIPSON, O.; LORD, A.; GUMUCIO, A.; O'CALLAGHAN, P.; LANNFELT, L.; NILSSON, L.N. Animal models of amyloid-beta-related pathologies in Alzheimer's disease. FEBS J., v.277, p.1389-409, 2010.

SCARMEAS, N.; LUCHSINGER, J.A.; SCHUPF, N.; BRICKMAN, A.M.; COSENTINO, S.; TANG, M.X.; STERN,Y. Physical Activity, Diet, and Risk of Alzheimer Disease. JAMA, v. 302, p. 627 637, 2009.

SCARMEAS, N.; STERN, Y.; MAYEUX, R.; MANLY, J.J.; SCHUPF, N.; LUCHSINGER, J.A. Mediterranean diet and mild cognitive impairment. Arch Neurol, v. 66, p. 216-225, 2009.

SCHAEFFER, E.L; FIGUEIRÓ, M.; GATTAZ,
W.F. Insights into Alzheimer disease pathogenesis from studies in transgenic animal models. Clinics, v. 66, p. 45-54, 2011.

SCHWARTMAN, R. A, CIDLOWSKI, J. A. Apoptosis: the biochemistry and molecular biology of programmed cell death. Endocrine Rev., v. 14, p. 133-51, 1993.

SHAMI NJ, MOREIRA EA. Licopeno como agente antioxidante. Rev. Nutr., v. 17 p. 227-236, 2004.

SHU, Y. AND CHECLER, F. Amyloid precursor protein, presenilins, and ?-synuclein: Molecular Pathogenesis and Pharmacological Application in Alzheimer's Diease. Pharmacol. Rev.. v. 54, p. 469-525, 2002.

SOZIO, P.; CERASA, L.S.; LASERRA, S.; CACCIATORE, I.; CORNACCHIA, C.; DI FILIPPO, E.S.; FULLE, S.; FONTANA, A.; DI CRESCENZO, A.; GRILLI, M.; MARCHI, M.; DI STEFANO, A. Memantine-sulfur containing antioxidant conjugates as potential prodrugs to improve the treatment of Alzheimer's disease. Eur J Pharm Sci. v. 49, p. 187-98, 2013.

STOCKER, R.; KEANEY, J.F, JR. Role of oxidative modifications in atherosclerosis. Physiol. Rev., v. 84, p. 1381-1478, 2004.

SULTANA, R.; PERLUIGI, M.; BUTTERFIELD, D. A. Protein oxidation and lipid peroxidation in brain of subjects with Alzheimer's disease: insights into mechanism of neurodegeneration from redox pro-teomics. Antioxid. Redox Signal, v. 8, p. 2021-2037, 2006.

VALKO, M.; LEIBFRITZ, D.; MONCOL, J.; CRONIN, M. T. D.; MAZUR, M.; TELSER, J. Free radicals and antioxidants in normal physiological functions and human disease. Int. J. Biochem. Cell Biol., v. 39, p. 44-84, 2007. 
VILA, M.; RAMONET, D.; PERIER, C.

Mitochondrial alterations in Parkinson's disease: new clues. J. Neurochem., v. 107, p. 317-328, 2008.

VINA, J.; LLORET, A.; ORTI, R.; ALONSO, D. Molecular bases of the treatment of Alzheimer's disease with antioxidants: prevention of oxidative stress. Mol. Aspects Med., v. 25, p. 117-123, 2004.

XIE, H.; HOU, S.; JIANG, J.; SEKUTOWICZ, M.; KELLY, J.; BACSKAI, B. J. Rapid cell death is preceded by amyloid plaque-mediated oxidative stress. Proc Natl Acad Sci USA., v. 7, p. 79047909, 2013. 